\title{
COMPETENCIAS EN FIN DE VIDA ENTRE UN GRUPO DE ENFERMEROS ESPAÑOLES Y CHILENOS
}

\section{NURSING COMPETENCES AT THE END OF LIFE AMONG A GROUP OF SPANISH AND CHILEAN NURSES}

María Oses Zubiri1 , Juan Manuel Casas Fernández de Tejerina², Fredy Seguel Palma3,

\section{${ }^{1}$ Enfermera Complejo Hospitalario de Navarra. Estudiante de doctorado UPNA. España}

2 Profesor Titular de la Universidad Pública de Navarra. Doctor en Medicina Interna. Complejo Hospitalario de Navarra. España

3 Profesor Auxiliar Instituto de Enfermería. Universidad Austral de Chile. Doctor en enfermería. Enfermero de referencia Hospital Base de Valdivia.

OSES ZUBIRI, M., Casas Fernández de Tejerina, J., \& Seguel Palma, F. (2020). COMPETENCIAS EN FIN DE VIDA ENTRE UN GRUPO DE ENFERMEROS ESPAÑOLES Y ChIlENos. Revista Ene De Enfermería, 14(2). Consultado de http://www.ene-enfermeria.org/ojs/index.php/ENE/article/view/1130 


\section{Resumen}

Evaluar las aptitudes de enfermería en la atención al final de la vida, nos permite percibir el nivel de cuidados proporcionado y mejorar su calidad.

Estudio observacional transversal, que utiliza la escala FATCOD-S en una muestra de enfermeros de Navarra (España) y Los Ríos (Chile).

Las enfermeras chilenas obtuvieron una mejor puntuación en la escala, respecto a las españolas. Se encontraron diferencias significativas según la edad, el contexto cultural y religioso, la formación y las experiencias previas de muerte. En este sentido, es necesario influir en aspectos relacionados con la comunicación, la toma de decisiones y el manejo de las emociones, para favorecer la satisfacción del cuidado terminal.

Palabras clave: actitudes al final de la vida; atención terminal; competencia clínica; competencia cultural; atención de enfermería;

\section{Abstract}

Assessing nursing skills in end-of-life care allows us to perceive the level of care provided and improve quality of care.

Cross-sectional observational study, which uses the FATCOD-S scale in a sample of nurses from Navarre (Spain) and Los Ríos (Chile).

Chilean nurses obtained a better score on the scale, compared to Spanish. Differences were found according to age, cultural and religious backgrounds, training and previous experiences of death. In this context, it is necessary to influence aspects related with communication, decision-making and emotion management, to favour the satisfaction of terminal care.

Key words: attitudes at the end of life; terminal care; clinical competence; cultural competence; nursing care; 


\section{INTRODUCCIÓN}

Se define "enfermedad terminal" como aquella en la que existe un proceso avanzado, incurable y progresivo, sin posibilidad razonable de respuesta a tratamientos específicos, y con un pronóstico de vida limitado a lo largo del tiempo (1). Según la OMS, los cuidados paliativos mejoran la calidad de vida de los pacientes y sus familias. Sin embargo, los profesionales de la salud siguen presentando obstáculos que limitan su autopercepción y capacitación en relación al cuidado teminal, afectando sobre todo en la accesibilidad a dichas atenciones (2).

Los factores determinantes que influyen en la asistencia de los cuidados paliativos, se pueden agrupar según el tipo de patología y el contexto en el que se desarrollan, y también de acuerdo al tipo de formación específica recibida por el personal de salud implicado. (3).

Alrededor del periodo de muerte de un individuo, se suceden una serie de características generales que tienen que ver con la reflexión objetiva del concepto de muerte, el control del dolor y el manejo adecuado de los síntomas presentes. Además, el reconocimiento civil de la dignidad de la persona moribunda, la presencia familiar y su apoyo en el cuidado, son aspectos igualmente importantes a considerar. Por todo ello y en base a estos parámetros, es necesario que exista una buena comunicación entre los profesionales de la salud, la familia y el propio paciente. ${ }^{(4)}$

En España, el $78 \%$ de las personas que mueren lo hacen en centros hospitalarios y en centros socio sanitarios (incluidos los hogares de ancianos) (5). Según un estudio realizado en las salas de urgencia de los hospitales, las enfermeras que manejaron las muertes de pacientes en fin de vida, consideraron que tenían barreras ambientales para proporcionar los cuidados paliativos adecuados, así como una formación en competencias deficiente para lidiar con el impacto emocional en dichas situaciones (6). En un intento por reducir las muertes hospitalarias, según aconseja la Estrategia Nacional de Cuidados Paliativos, las enfermeras españolas "gestoras de casos" encuentran difícil la coordinación entre niveles atención primaria y atención especializada, determinada principalmente por la falta de información y planificación al alta ante el traslado de los pacientes terminales a sus domicilios (7) (8). Si nos situamos en otro contexto geográfico como es Chile, varias investigaciones demuestran que las enfermeras desarrollan una actitud de compromiso y liderazgo, de acuerdo también con el Plan Nacional de Alivio del Dolor y Cuidados Paliativos. Según este programa, brindar atención espiritual mejora 
la sensación de serenidad y paz, lo que hace que la fase final de la vida sea menos vulnerable y más llevadera, tanto para los pacientes como para los miembros de la familia (9).

En ese sentido, los aspectos relacionados con las competencias en las que se apoya la atención de enfermería paliativa, van dirigidas a desarrollar un proceso de atención desde la perspectiva multidisciplinaria, así como a una adecuada adquisición de conocimientos sobre gestión farmacológica, psicológica y socio legal (10). Además, la empatía y la comunicación asertivas facilitan el papel de las enfermeras en este marco de actuación, ya que mejoran la sensación de soledad en los pacientes y en sus familias y reducen la ansiedad y el agotamiento mental, lo que viene reflejado en una mayor satisfacción vital para ambos (11).

El término competencia entendido como habilidad, tiene que ver con la "disposición para el buen desempeño o el ejercicio de un arte". En este caso, la actitud, el rendimiento o la capacidad se definen como el carácter o el conjunto de condiciones que hacen que una persona sea particularmente adecuada para una función específica (12). La integración emocional de las habilidades de enfermería en los cuidados paliativos, sigue un proceso de transformación que, en cualquier caso, se divide en tres fases, según el estudio realizado por Huang et al. Inicialmente, hay un sufrimiento espiritual en el profesional provocado por la muerte del paciente, seguido de una segunda fase en la que tanto el enfermero como el equipo de trabajo generan diversas estrategias de afrontamiento. Finalmente, se desarrolla un tercer nivel de reflexión y aprendizaje en el individuo, que se refleja ante la responsabilidad de ofrecer los mejores cuidados disponibles bien para el paciente como para las familias (13).

Llegados a este punto, podemos añadir que la cultura y el grado de integración de los programas de cuidados paliativos, sea de forma estratégica como territorial, es lo que diferencia la calidad de la asistencia ofrecida en todos los niveles de salud involucrados. La promoción de estos aspectos en la sociedad, el uso de herramientas pedagógicas innovadoras y una mayor participación en el plano político, especialmente en el fomento de medidas a nivel ambulatorio, son las principales recomendaciones que mejorarían actualmente el acceso a los servicios paliativos (14).

Entre las herramientas disponibles en la literatura, para evaluar las aptitudes en la atención al paciente terminal, encontramos la escala diseñada por K. Frommelt en 2003 (FATCOD-B), también utili- 

COMPETENCIAS EN FIN DE VIDA ENTRE UN GRUPO DE ENFERMEROS ESPAÑOLES Y CHILENOS

zada para evaluar un programa cualquiera de formación en cuidados paliativos (15). Esta herramienta ha sido traducida y validada en varios idiomas, incluidos el español, el italiano, el japonés, el sueco, el chino, etc. Según el análisis factorial realizado en el contexto español por Edo Gual et al. (FATCOD-S), existen cuatro modelos de medición que se derivan de esta escala (16):

-el modelo unidimensional FrommeltA (escala inicial de 1991) (17)

-el modelo de dos factores descrito por Nakai, que agrupa los componentes en el factor tipo I, en relación con las actitudes positivas hacia la atención del paciente terminal, y en el factor tipo II, que tiene que ver con las percepciones del profesional sanitario sobre la atención centrada en el paciente y la familia (18) (19)

- el modelo propuesto por Henoch, donde $2 / 3$ del cuestionario se refiere a las actitudes hacia el cuidado de pacientes con enfermedades terminales y $1 / 3$ son cuestiones relacionadas sobre el cuidado de la familia (20).

- y el modelo de cuatro componentes citado por Leombruni, que divide la escala en factor I-compromiso emocional, factor II-creencias sobre la asistencia al final de la vida, factor III-creencias sobre los límites personales y factor IV-creencias y sentimientos sobre muerte (21).
Los objetivos de este estudio, según las características de la investigación, son evaluar las habilidades de enfermería en el cuidado del paciente terminal y sus familiares, utilizando la escala FATCOD-S traducida al español. De este modo se pretende determinar, la relación de las competencias descritas en la escala según distintas variables sociodemográficas como pueden ser el lugar de origen, la edad, la experiencia profesional, el sexo, el estado civil, la religión, la asistencia a funerales durante la infancia, la experiencia de pérdida de un ser querido y las percepciones emocional y técnica de la atención al paciente terminal.

\section{MÉTODO}

Estudio descriptivo transversal, que explora las habilidades de enfermería en el cuidado del paciente terminal y su familia. Los datos se obtuvieron por muestreo de conveniencia durante 2014 y 2015, hasta alcanzar 439 participantes, en las provincias de Navarra (España) y de Los Ríos (Chile). Para su desarrollo, se solicitó la evaluación de los comités de ética de la Universidad Pública de Navarra y del Departamento de Salud de Valdivia (Chile) respectivamente. Los criterios de inclusión en el estudio fueron ser enfermeros/as en activo de organismos públicos y privados en las zonas 

COMPETENCIAS EN FIN DE VIDA ENTRE UN GRUPO DE ENFERMEROS ESPAÑOLES Y CHILENOS

seleccionadas, y en particular de cuatro hospitales regionales, dos de cada área geográfica (en los que participaron departamentos médicos, quirúrgicos y de urgencias) y un total de 26 centros de salud ambulatorios.

Se administró un cuestionario a cada participante en el lugar de trabajo, que inicialmente contenía una explicación de los objetivos del estudio, un cuestionario sobre variables sociodemográficas $\mathrm{y}$, por último, la escala FATCOD traducida al español (Anexo l), cuyo uso fue expresamente autorizado por la autora K. Frommelt. Esta escala se compone de 30 elementos tipo Likert de 1 punto (totalmente en desacuerdo), 3 puntos (neutral) y 5 puntos (totalmente de acuerdo) respectivamente. Se empleó el programa SPSS 20.0 (IBM Corp.) para realizar el análisis estadístico. La validación interna de la escala se calculó a través del a de Cronbach. Por otro lado, se siguió el modelo propuesto por Edo Gual, que elimina el elemento 10 del cuestionario ("A veces la muerte es aceptada por la persona que se está muriendo") y agrupa el resto de los elementos en dos factores: el factor I, relacionado con la percepción de las actitudes positivas hacia el cuidado de pacientes con enfermedades terminales (compuesto por los elementos $1,2,3,5,6,7,8,9,11,13,14$, $15,17,26,29$ y 30 ) y el factor II, que describe la percepción de los cuidados centrados en el paciente terminal y su familia (descrito por los elementos 4, 12, $16,18,19,20,21,22,23,24,25,27$ y 28). Se han utilizado pruebas no paramétricas Mann-Withney y Kruskall Wallis para evaluar la correlación entre las variables sociodemográficas y la escala.

\section{REsultados}

Se entregaron un total de 700 cuestionarios, de los cuales 325 se consideraron válidos en Navarra y 114 en Los Ríos, obteniendo una participación aproximada del $63 \%$.

El resultado del alfa de Cronbach fue cuestionable en ambos grupos, 0.524 en Navarra y 0.617 en Los Ríos, y no se modificaba demasiado si se eliminaba el ítem 10 como aconsejaban los autores (a 0.523 y 0.625 respectivamente) (16). Una evaluación del mismo instrumento, realizada por Mastroianni, mostró un alfa de Cronbach de 0.83 (22). Sin embargo, en el estudio realizado por Henoch, el valor también fue bajo (a 0.506) (23).

En ambos grupos, la actitud hacia la atención del paciente terminal es positiva en un porcentaje similar $(p<0.246)$ (tabla 1).

Como se evidencia en la Tabla 2, las enfermeras chilenas obtuvieron una puntuación significativamente más alta de la 

COMPETENCIAS EN FIN DE VIDA ENTRE UN GRUPO DE ENFERMEROS ESPAÑOLES Y CHILENOS

escala con respecto a las enfermeras españolas ( $p<0.002)$. Por otro lado, el porcentaje de mujeres fue claramente mayor que el de hombres, aunque no se obtuvieron diferencias significativas al respecto ( $p<0,558)$. Se obtuvo una puntuación más alta en el grupo de enfermeras de menor edad y menos años de experiencia profesional ( $p<0,000$ ), así como en aquellos que trabajaban en el entorno hospitalario en comparación con el ambulatorio ( $p<0,002)$ y en aquellas enfermeras cuya residencia se situaba en un entorno rural ( $p<0.001$ ). Aquellos que dicen profesar una religión, alcanzaron una puntuación más alta ( $p<0.001)$, al igual que aquellos que no asistían a funerales en su infancia o no habían padecido la pérdida de un ser querido $(p$ $<0.05)$. Finalmente, aquellos con mejor percepción emocional para el manejo de la atención de pacientes terminales, obtuvieron mejores cifras en la escala $(p$ $<0.001$ ).

Teniendo en cuenta la puntuación de cada enunciado, el ítem 21 ("Es beneficioso para el moribundo verbalizar sus sentimientos ") es el que obtiene mayor puntuación de toda la escala en ambos grupos; mientras que el ítem 19 (“El moribundo no tiene permitido tomar decisiones sobre su cuidado físico), es el que obtiene la puntuación más baja.
Con respecto al factor I, sobre las actitudes positivas del profesional hacia la asistencia a pacientes terminales, encontramos diferencias significativas según el lugar de origen como se muestra en la tabla 3 . Las enfermeras chilenas obtienen unos valores de media más altos en el ítem 1 ("Proporcionar cuidados al moribundo es una experiencia que merece la pena ") ( $p<0.025)$, en el ítem 2 ("La muerte no es lo peor que le puede pasar a una persona") ( $p<0.007$ ), el ítem 7 (“El periodo de tiempo requerido para atender a un moribundo me frustraría ") ( $p$ $<0.012$ ), el ítem 11 ("Cuando un paciente pregunta ¿me estoy muriendo? creo que es mejor cambiar de tema hacia algo más alegre") $(p<0.001)$, el ítem 13 ("Tendría esperanzas de que la persona a la que atiendo muriera cuando no esté") ( $p<0.022$ ) y el ítem 17 ("Conforme el paciente se aproxima a la muerte, los cuidadores ajenos a la familia deben retirar su implicación con el paciente") ( $p$ $<0.024)$. Al contrario, las enfermeras españolas obtienen una puntuación mayor en el ítem 5 ("A mí no me gustaría atender a un moribundo ") ( $p<0.021)$ y en el ítem 15 ("Sentiría ganas de huir cuando la persona por fin muriera") ( $p<0,008)$.

Según el factor II, en relación con la percepción de actitudes sobre los cuidados centrados en el paciente terminal y su familia, las enfermeras de Los Ríos 

COMPETENCIAS EN FIN DE VIDA ENTRE UN GRUPO DE ENFERMEROS ESPAÑOLES Y CHILENOS

alcanzaron una puntuación mayor en el ítem 4 ("Las atenciones a la familia del paciente deberían continuar a lo largo del periodo de dolor y duelo") ( $p<0.000)$, el ítem 12 ("La familia debería estar involucrada en el cuidado físico del moribundo") ( $p<0.000$ ), el ítem 16 ("Las familias necesitan un soporte emocional para aceptar los cambios de humor del moribundo") ( $p<0.000$ ), el ítem 20 ("Las familias deben mantener un ambiente tan normal como sea posible para el moribundo") ( $p<0.000$ ), el ítem 21 ("Es beneficioso para el moribundo verbalizar sus sentimientos") ( $p<0.000$ ), el ítem 22 ("Los cuidados deben extenderse hasta los familiares del moribundo") ( $p<0.000$ ), el ítem 23 ("Los cuidadores deben ofrecer a los moribundos un horario flexible de visitas") ( $p<0.003$ ), el ítem 24 ("El moribundo y su familia deben ser los responsables de la toma de decisiones") ( $p<0.008$ ) y el ítem 27 ("Los moribundos deben recibir respuestas sinceras sobre su enfermedad ") ( $p<0.002)$. Por otro lado, las enfermeras de Navarra obtuvieron puntuaciones más altas en el ítem 25 ("La adicción a la medicación contra el dolor no debe preocupar cuando se trata de un moribundo ") $(p<0.008)$ como se describe en la tabla 4.

El análisis realizado entre las variables sociodemográficas y el cuestionario de Frommelt se describe en la tabla 5.
Se hallaron diferencias significativas para el sexo femenino en el ítem 16 ( $p$ $<0.018$ ) y en relación con los que trabajaban en el hospital en los ítems 11 y 23 respectivamente $(p<0,05)$. En cuanto a la situación familiar, los que se encontraban entre "otras situaciones familiares" (viudos/as, divorciados/as, solteros/as, etc.), obtuvieron una puntuación mayor en el ítem 4 ( $p<0.054)$, mientras que aquellos con pareja y con/sin hijos puntuaron más alto en el ítem 7 ( $p$ $<0.024)$. Para las enfermeras residentes en áreas rurales, el valor del ítem 3 fue significativamente más alto ( $p<0.017$ ), en comparación con las residentes en áreas urbanas, las cuales obtuvieron una mayor puntuación en el ítem 16 ( $p$ $<0.049$ ). Por otro lado, aquellos que profesaban una religión, obtuvieron una puntuación más alta en los ítems del factor II ( $p<0.024)$, en comparación con aquellos que decían no profesar, quienes obtuvieron una puntuación más alta en el ítem 2 ( $p$ <0.023). Los que practicaban la religión 1 / semana, obtuvieron una puntuación más alta en los ítems 8 y 19 ( $p$ $<0.05)$ y, los que lo hacían 1 / mes, obtuvieron una puntuación más significativa en los ítems 22 y 30 ( $p<0,05)$. Las enfermeras que asistían a funerales en la infancia, obtuvieron más alto en el ítem 6 ( $p<0.009$ ). Del mismo modo, aquellos con una experiencia previa de pérdida de 
un ser querido, obtuvieron mayor puntuación en los ítems 23 y 25 ( $p<0.05)$, frente a las enfermeras que no sufrieron la experiencia de pérdida, quienes alcanzaron más valor en el ítem 2 ( $p$ $<0.052)$. En relación con los grupos de edad, se distinguieron entre las enfermeras más jóvenes (24-34 años), que obtuvieron más puntuación en los ítems 11, 13 y 18 ( $p<0.05$ ). En cambio, los adultos jóvenes (35-44 años) alcanzaron mayor puntuación en los ítems 16 y 23 ( $p$ $<0.05)$ frente aquellos con una edad intermedia (45-54 años), los cuales obtuvieron más puntuación en los ítems 3 y 5 ( $p$ <0.05). Los enfermeros de mayor edad (55-65 años), obtuvieron puntuaciones más altas en los ítems 19 y 25 ( $p$ $<0.05)$ respectivamente.

En cuanto a la experiencia profesional, se encontró una puntuación más alta dentro del grupo con menos años de experiencia laboral ( $<5$ años) en los ítems $4,11,12,13,16$ y 18 ( $p<0.05)$. Además, se encontraron diferencias significativas en el ítem 23 para el grupo de 15-26 años de experiencia, y también una puntuación más alta en el ítem 25 entre aquellos con más experiencia profesional (<25 años). Finalmente, las enfermeras con 1-3 puntos sobre el grado de percepción con respecto a la preparación emocional y técnica para administrar cuidados al final de la vida, obtuvieron una puntuación más alta en la escala ( $p$ $<0,000)$.

\section{DISCUSIÓN}

Dentro de las competencias que debe manejar un profesional de enfermería, entendemos que todas ellas deben favorecer las decisiones sobre el proceso de enfermedad y muerte. Según una investigación al respecto, el ambiente de trabajo y el nivel de educación son factores determinantes que aumentan la capacidad de participación de las enfermeras en este sentido (24).

En numerosas ocasiones los profesionales de la salud prefieren no afrontar la atención de pacientes en estado terminal, y evitan estar en contacto con dichas situaciones por temor a no poder manejarlas, o también debido a una falta de recursos personales y habilidades de comunicación al respecto (25). Como se evidencia en el estudio desarrollado por Kirkpatrick et al., la educación universitaria es importante en la adquisición de habilidades de enfermería en atención al final de la vida; coincide pues con el presente estudio, donde se refleja un mayor nivel de competencias entre aquellos que han recibido mayor carga lectiva a nivel de grado (26). Aunque los resultados obtenidos de ambos grupos participantes son favorables con respecto a la atención del paciente terminal, la puntuación 

COMPETENCIAS EN FIN DE VIDA ENTRE UN GRUPO DE ENFERMEROS ESPAÑOLES Y CHILENOS

total de la escala es significativamente menor que la obtenida en otros estudios como el realizado por Leombruni en Italia (M 115.2 (Ds 7.86)) (21), el conducido por Henoch en Suecia (M 126.3 (Ds 5.9)) (23), o el elaborado por Espinoza-Venegas en Chile (M 124.23 (Ds 10.2)) (27). Esto justifica la necesidad de llevar a cabo programas de mejora de la capacitación de habilidades del cuidado en fin de vida en ambos contextos de Navarra y Los Ríos.

Muchas investigaciones han utilizado la escala K. Frommelt para evaluar las competencias de enfermería en el cuidado del paciente con enfermedad terminal y a su familia (28) (29). Un ejemplo de ello es el estudio realizado en el contexto español, que determina que los estudiantes de enfermería con experiencias previas de muerte y los que recibieron formación en cuidados paliativos, obtuvieron una mejor puntuación en la escala (16). Por otro lado, otras averiguaciones han descrito que, aquellas enfermeras de mayor edad, que pasan más tiempo con personas moribundas, presentan una mejor actitud hacia su cuidado, frente a las que trabajan en otros entornos laborales (23).

La cultura y la religión juegan un papel importante en el desarrollo del concepto de muerte y su manejo emocional. Es importante adquirir capacidades rela- cionadas con la religión y haber tenido experiencias previas de muerte, para facilitar la atención adecuada del paciente paliativo y sus familias (30). Del mismo modo, la correcta percepción de uno mismo hacia la muerte del paciente, lleva a conmemorar pérdidas personales pasadas y favorece el aumento de estrategias de afrontamiento relacionadas con un sufrimiento espiritual en el profesional sanitario (31). Por otro lado, la participación en funerales según las creencias culturales, y también la posibilidad de despedirse o recordar a las personas que murieron en un entorno natural, contribuye al crecimiento personal que da sentido y profundiza en la reflexión sobre la pérdida del ser querido (32). Es necesario tener en cuenta además otros aspectos como la edad, el lugar de residencia o el género al examinar las actitudes de los cuidados de enfermería al final de la vida (33). En cuanto al uso de opioides, este aumenta en un $60-80 \%$ durante los últimos 5 meses de vida, como se destaca en el estudio realizado a nivel europeo por Paque et al (34), por ello siempre debemos tener en cuenta la posibilidad de una mortalidad temprana asociada con su uso inadecuado, principalmente debido a los efectos secundarios que éstos pueden causar (35).

Además, un estudio reciente realizado a través de una entrevista semiestructu- 

COMPETENCIAS EN FIN DE VIDA ENTRE UN GRUPO DE ENFERMEROS ESPAÑOLES Y CHILENOS

rada con enfermeras de $\mathrm{UCl}$, ha demostrado que la reflexión profesional y el codesarrollo conjunto, reducen el estrés y mejoran las habilidades de quienes brindan cuidados a pacientes con enfermedades terminales. En este caso, el liderazgo es una herramienta importante para promover actitudes que mejoren la calidad de los cuidados paliativos, especialmente en las enfermeras de primer nivel (36).

\section{CONCLUSIÓN}

Mediante la realización del presente estudio, se pretende concienciar entre los profesionales de la enfermería, la importancia de practicar un adecuado manejo de las habilidades que conforman el cuidado de personas que se encuentran en estados avanzados de enfermedad, entre los que hay que tener en cuenta además a la unidad familiar como parte importante del binomio paciente-cuidador. A través del empleo de la escala de Frommelt, se ha evidenciado la presencia de una alta carga emocional asociada al proceso de duelo y muerte, que el profesional de enfermería experimenta en función de sus características sociodemográficas. Entre ellas encontramos diversos factores como los relacionados con la edad, la formación en cuidados paliativos, la cultura, la religión o las experiencias previas de muerte. Propone- mos continuar el estudio de las actitudes de enfermería al final de la vida como parte de la mejora continua de los cuidados que se han de aplicar en la práctica diaria, y como parte de los programas de formación adaptados para ello.

\section{AGRADECIMIENTOS}

Se agradece la colaboración de la Universidad Austral de Chile por favorecer la estancia formativa para el trabajo de campo realizado en Valdivia (Región de Los ríos en Chile).

Se agradece el interés mostrado por todos los enfermeros participantes en el estudio, así como a las personas gestoras que han ayudado en su desarrollo.

Por último agradecer el consentimiento de la autora K Frommelt para otorgar el uso de la escala diseñada por ella en esta investigación. 


\section{BiBLIOGRAFÍA}

1.SECPAL. Sociedad Española de cuidados paliativos. [Online].; 2014 [cited 2016 Nov 3. Disponible en: https://www.secpal.com/guia-cuidados-paliativos1

2.OMS. Cuidados paliativos. Notas descriptivas. [Online].; 2019 [cited 2019 Octubre 9. Disponible en: https://www.who.int/es/news-room/fact-sheets/detail/ palliative-care

3.Osés Zubiri M, Casas Fernández J. Factors influencing the conceptualization in palliative care. 2017. Poster: P01-265; 15th World Congress of the European Association for palliative care. Madrid.

4.Granda-Cameron C, Houldin A. Concept analysis of good death in terminally ill patients. Am J Hosp Palliat Care. 2012; 29(8): p. 632-9.

5.Urmeneta A. Derecho a morir dignamente en España. [Online].; 2015 [cited 2015 May 25. Disponible en: http://www.eutanasia.ws/hemeroteca/t169.pdf.

6.Giles T, Hammad K, Breaden K, Drummond C, Bradley S, Gerace A, et al. Nurses' perceptions and experiences of caring for patients who die in the emergency department setting. Int Emerg Nurs. 2019 Sep; 5 .

7.Correa-Casado M, Granero-Molina J, Hernández-Padilla J, Fernández-Sola $\mathrm{C}$. Transferring palliative-care patients from hospital to community care: A qualitative study. Aten primaria. 2017 Jun-Jul; 49(6): p. 326-334

8.Ministerio de Sanidad Política social e Igualdad. Estrategia en Cuidados Paliativos del Sistema Nacional de Salud. Actualización 2010-2014. Madrid: Gobierno de España; 2011.

9.Contreras Contreras S, Sanhueza Alvarado O. Patients of the Pain relief and palliative care programme: reasons and meaning for nurses. Cienc. enferm. $2016 \mathrm{abr}$; 22(1).

10.Ferrell B, Malloy P, Mazanec P, Virani R. CARES: AACN's New competencies and recommendations for educating undergraduate nursing students to improve palliative care. J Prof Nurs. 2016 Sep-Oct; 32(5): p. 327-33.

11.Marilaf Caro M, San-Martín M, Delgado-Bolton R, Vivanco L. Empathy, loneliness, burnout, and life satisfaction in Chilean nurses of palliative care and homecare services. Enferm Clin. 2017 Nov-Dec; 27(6): p. 379-386.

12.Real Academia Española. Diccionario de la lengua española. [Online].; 2014 [cited 2017 Marzo 8. Disponible en: http://dle.rae.es/?id=3KcD1v9.

13. Huang $\mathrm{C}$, Chen J, Chiang $\mathrm{H}$. The transformation process in nurses caring for dying patients. $J$ Nurs Res. 2016 Jun; 24(2): p. 109-17.

14.Brant J, Fink R, Thompson C, Li Y, Rassouli $M$, Majima $T$, et al. Global survey of the roles, satisfaction, and barriers of home health care nurses on the provision of palliative care. J Palliat Med. 2019 Aug; 22(8): p. 945-960.

15.Frommelt K. Attitudes toward care of the terminally ill: an educational intervention. American Journal of Hospice \& Palliative Care. 2003; 20(1): p. 13-22.

16.Edo-Gual M, Tomás-Sábado, Gómez-Benito J, C MR, Aradilla-Herrero A. Spanish adaptation of the Frommelt Attitudes toward caring of the dying scale (FATCOD-S) in nursing undergraduates. OMEGAJournal of death and dying. 2017; 0(0): p. 1-23.

17.Frommelt $\mathrm{K}$. The effects of death education on nurse's attitudes toward caring for terminal ill persons and their families. Am J Hosp Palliat Care. 1991 SepOct; 8(5): p. 37-43.

18.Nakai $\mathrm{Y}$, Miyashita M, Sasahara T, Koyama $\mathrm{Y}$, Shimizu Y, Kawa M. Factor structure and reliability of the Japanese version of the Frommelt attitude toward care of dying scale. Japanese Journal of Cancer Nursing. 2006;(11): p. 723-29.

19.Miyashita M, Nakai Y, Sasahara T, Koyama Y, Shimizu $\mathrm{Y}$, Tsukamoto N, et al. Nursing autonomy plays an important role in nurses' attitudes toward caring for dying patients. Am J Hosp Palliat Care. 2007 Jun-Jul; 24(3): p. 202-10.

20. Henoch I, Browall M, Melin-Johansson C, Danielson E, Udo C, Johansson Sundler A, et al. The Swedish version of the Frommelt Attitude Toward Care of the Dying scale: aspects of validity and factors influencing nurses' and nursing students' attitudes. Cancer Nurs. 2014 Jan-Feb; 37(1): p. E1-11.

21.Leombruni P, Miniotti M, Bovero A, Zizzi F, Castelli L, Torta R. Attitudes toward caring for dying patients: An overview among Italian nursing students and preliminary psychometrics of the FATCOD-B scale. Journal of Nursing Education and Practice. 2014; 4(3): p. 188-96.

22.Mastroianni C, Piredda M, Frommelt K, Mirabella $F$, Taboga $C$, Casale $\mathrm{G}$, et al. Italian validation of the Frommelt attitudes toward care of the dying Scale form B (FATCOD Form B-I). Int Nurs Perpect. 2009; 9(1): p. 11-6.

23. Henoch I, Browall M, Melin-Johansson C, Danielson E, Udo C, Johansson Sundler A, et al. The Swedish version of the Frommelt Attitude Toward Care of the Dying scale: aspects of validity and factors influencing nurses' and nursing students' attitudes. Cancer Nurs. 2014; 37(1): p. E1-11.

24.Albers G, Francke AL, de Veer A, Bilsen J, Onwuteaka-Philipsen B. Attitudes of nursing staff towards involvement in medical end-of-life decisions: A national survey study. Patient Educ Couns. 2014 Jan; 94(1): p. 4-9.

25.Dobrowolska B, Mazur E, Pilewska-Kozak A, Dońka K, Kosicka B, Palese A. Predicted difficulties, educational needs, and interest in working in end of life care among nursing and medical students. Nurse Educ Today. 2019 Dic; 83(104194). 
26.Kirkpatrick A, Cantrell M, Smeltzer S. Relationships among nursing student palliative care knowledge, experience, self-awareness, and performance: An end-of-life simulation study. Nurse Educ Today. 2019 Feb; 73: p. 23-30.

27.Espinoza Venegas M, Luengo Machuca L, Sanhueza Alvarado O. Attitudes held by Chilean nursing professionals on care of the dying. A multivariate analysis. Aquichan. 2016; 16(4): p. 430-446.

28. Morita T, Murata $\mathrm{H}$, Hirai K, Tamura K, Kataoka J, Ohnishi $\mathrm{H}$, et al. Meaninglessness in terminally ill cancer patients: a validation study and nurse education intervention trial. J Pain Symptom Manage. 2007; 34(2): p. 160-70.

29.Watson Alvaro A. Critical care nurse's attitudes towards the care of the dying: an educational intervention. Degree of Master of Science in Nursing. Western Carolina University, Nursing Department; 2009

30.Liping W, Jing C, Yanli D, Zhenzhen W, Zhifang L, Zhaoyang D. Factors Influencing Chinese nursing Students' Attitudes Toward the Care of Dying Patients. Journal of Hospice \& Palliative Nursing. 2017 Aug; 19 (4): p. 343-350.

31.Garrino L, Contratto C, Massariallo P, Dimonte V. Caring for dying patient and their families: the lived experiences of nursing students in Italy. Journal of palliative Care. 2017 Nov; 32(3-4): p. 127-133.

32.Draper P, Holloway M, Adamson S. A qualitative study of recently bereaved people's beliefs about death: implications for bereavement care. J Clin Nurs. 2014 May; 23(9-10): p. 1300-8.

33. Hagelin C, Melin-Johansson C, Henoch I, Bergh I, Ek K, Hammarlund K, et al. Factors influencing attitude toward care of dying patients in first-year nursing students. Int J Palliat Nurs. 2016 Jan; 22(1): p. 28-36.

34. Paque K, Elseviers M, Vander Stichele R, Pardon K, Hjermstad MJ, Kaasa S, Dilles T, De Laat $\mathrm{M}$, Van Belle S, Christiaens T, Deliens L. Changes in medication use in a cohort of patients with advanced cancer: The international multicentre prospective European Palliative Care Cancer Symptom study. Palliat Med. 2018 Apr; 32(4): p. 775-785.

35.Álvarez Mazariegos, JA; Calvete Waldomar, S; Fernández-Marcote Sánchez-Mayoral, RM; Guardia Serecigni, J. Guía de consenso para el buen uso de analgésicos opioides. Gestión de riesgos y beneficios. Valencia: SECPAL-SEMFyC-FAECAP; 2017.

36. White D, Meeker M. Guiding the process of dying: the personal impact on nurses. J Hosp Palliat Nurs (Publish Ahead of Print). 2019 Oct; 21(5): p. 390-396. 


\section{FIGURAS, TABLAS Y ANEXOS}

Anexo I. Versión española de la escala FATCOD (FATCOD-S)

\begin{tabular}{|c|c|c|c|c|c|}
\hline $\begin{array}{l}\text { Escala de Frommelt sobre actitudes ante los cuidados del paciente } \\
\text { moribundo. }\end{array}$ & $\begin{array}{l}\text { Totalmente en } \\
\text { desacuerdo }\end{array}$ & $\begin{array}{l}\text { De } \\
\text { acuerdo }\end{array}$ & Neutro & $\begin{array}{l}\text { De } \\
\text { acuerdo }\end{array}$ & $\begin{array}{l}\text { Totalmente de } \\
\text { acuerdo }\end{array}$ \\
\hline $\begin{array}{l}\text { 1.Proporcionar cuidados de enfermería a una persona que se está } \\
\text { muriendo es una experiencia muy valiosa }\end{array}$ & 1 & 2 & 3 & 4 & 5 \\
\hline 2.La muerte no es lo peor que le puede suceder a una persona & 1 & 2 & 3 & 4 & 5 \\
\hline $\begin{array}{l}\text { 3.Me resultaría incómodo hablar con una persona que se está } \\
\text { muriendo de su propia muerte }\end{array}$ & 1 & 2 & 3 & 4 & 5 \\
\hline $\begin{array}{l}\text { 4.Los cuidados de enfermería a la familia deben continuar durante } \\
\text { todo el proceso de duelo }\end{array}$ & 1 & 2 & 3 & 4 & 5 \\
\hline $\begin{array}{l}\text { 5.No me gustaría tener que cuidar a una persona que se esté } \\
\text { muriendo }\end{array}$ & 1 & 2 & 3 & 4 & 5 \\
\hline $\begin{array}{l}\text { 6. La enfermera no es la persona adecuada para hablar de la muerte } \\
\text { con una persona que se esté muriendo }\end{array}$ & 1 & 2 & 3 & 4 & 5 \\
\hline $\begin{array}{l}\text { 7.Me provocaría frustración el tiempo que requiere proporcionar } \\
\text { cuidados de enfermería a una persona que se esté muriendo }\end{array}$ & 1 & 2 & 3 & 4 & 5 \\
\hline $\begin{array}{l}\text { 8.Me molestaría que una persona moribunda a la que estuviera } \\
\text { cuidando perdiera la esperanza de mejorar }\end{array}$ & 1 & 2 & 3 & 4 & 5 \\
\hline $\begin{array}{l}\text { 9.Es difícil establecer una relación estrecha con la familia de una } \\
\text { persona que se está muriendo }\end{array}$ & 1 & 2 & 3 & 4 & 5 \\
\hline $\begin{array}{l}\text { 10.A veces la muerte es aceptada por la persona que se está } \\
\text { muriendo }\end{array}$ & 1 & 2 & 3 & 4 & 5 \\
\hline $\begin{array}{l}\text { 11. Cuando un paciente pregunta a la enfermera ¿me estoy } \\
\text { muriendo? Pienso que es mejor cambiar de tema }\end{array}$ & 1 & 2 & 3 & 4 & 5 \\
\hline $\begin{array}{l}\text { 12.La familia debería implicarse en los cuidados físicos de la persona } \\
\text { que se está muriendo }\end{array}$ & 1 & 2 & 3 & 4 & 5 \\
\hline $\begin{array}{l}\text { 13.Preferiría que la persona a la que estoy cuidando muriese cuando } \\
\text { yo no esté }\end{array}$ & 1 & 2 & 3 & 4 & 5 \\
\hline $\begin{array}{l}\text { 14.Me da miedo establecer una amistad con la persona que se está } \\
\text { muriendo }\end{array}$ & 1 & 2 & 3 & 4 & 5 \\
\hline 15.Cuando una persona se muere, me dan ganas de salir corriendo & 1 & 2 & 3 & 4 & 5 \\
\hline $\begin{array}{l}\text { 16. La familia necesita un soporte emocional para aceptar los cambios } \\
\text { en la conducta de la persona que se está muriendo }\end{array}$ & 1 & 2 & 3 & 4 & 5 \\
\hline $\begin{array}{l}\text { 17. Cuando un paciente está cercano a la muerte, la enfermera } \\
\text { debería dejar de implicarse en su cuidado }\end{array}$ & 1 & 2 & 3 & 4 & 5 \\
\hline $\begin{array}{l}\text { 18.La familia debe preocuparse de que el enfermo viva lo mejor } \\
\text { posible lo que le queda de vida }\end{array}$ & 1 & 2 & 3 & 4 & 5 \\
\hline $\begin{array}{l}\text { 19.La persona que se está muriendo no debería tomar decisiones } \\
\text { sobre sus cuidados físicos }\end{array}$ & 1 & 2 & 3 & 4 & 5 \\
\hline $\begin{array}{l}\text { 20.La familia debería mantener un entorno tan normal como sea } \\
\text { posible por el bien del enfermo }\end{array}$ & 1 & 2 & 3 & 4 & 5 \\
\hline $\begin{array}{l}\text { 21.Es bueno que la persona que se está muriendo verbalice sus } \\
\text { sentimientos }\end{array}$ & 1 & 2 & 3 & 4 & 5 \\
\hline $\begin{array}{l}\text { 22. Los cuidados de enfermería deben hacerse extensivos a la familia } \\
\text { de la persona que se está muriendo }\end{array}$ & 1 & 2 & 3 & 4 & 5 \\
\hline $\begin{array}{l}\text { 23. Las enfermeras deben permitir un horario flexible de visitas para } \\
\text { las personas que se están muriendo }\end{array}$ & 1 & 2 & 3 & 4 & 5 \\
\hline $\begin{array}{l}\text { 24.La persona que se está muriendo y su familia deberían ser los } \\
\text { responsables de la toma de decisiones }\end{array}$ & 1 & 2 & 3 & 4 & 5 \\
\hline $\begin{array}{l}\text { 25.La adicción a los analgésicos no debe ser una preocupación para } \\
\text { las enfermeras de un paciente moribundo }\end{array}$ & 1 & 2 & 3 & 4 & 5 \\
\hline $\begin{array}{l}\text { 26. Me sentiría incómodo si al entrar en la habitación de un paciente } \\
\text { con una enfermedad terminal, me lo encontrase llorando }\end{array}$ & 1 & 2 & 3 & 4 & 5 \\
\hline $\begin{array}{l}\text { 27.Las personas moribundas deberían recibir respuestas honestas } \\
\text { sobre su estado }\end{array}$ & 1 & 2 & 3 & 4 & 5 \\
\hline
\end{tabular}




\begin{tabular}{|c|c|c|c|c|c|}
\hline $\begin{array}{l}\text { 28.Educar a las familias sobre la muerte y el proceso de morir no es } \\
\text { una responsabilidad de enfermería }\end{array}$ & 1 & 2 & 3 & 4 & 5 \\
\hline $\begin{array}{l}\text { 29.Los familiares que permanecen junto al paciente moribundo, a } \\
\text { menudo interfieren en el trabajo del profesional con el paciente }\end{array}$ & 1 & 2 & 3 & 4 & 5 \\
\hline $\begin{array}{l}\text { 30.Las enfermeras pueden ayudar a los pacientes a prepararse para } \\
\text { la muerte }\end{array}$ & 1 & 2 & 3 & 4 & 5 \\
\hline
\end{tabular}

Figura 1. Factores determinantes en la atención al paciente terminal y su familia

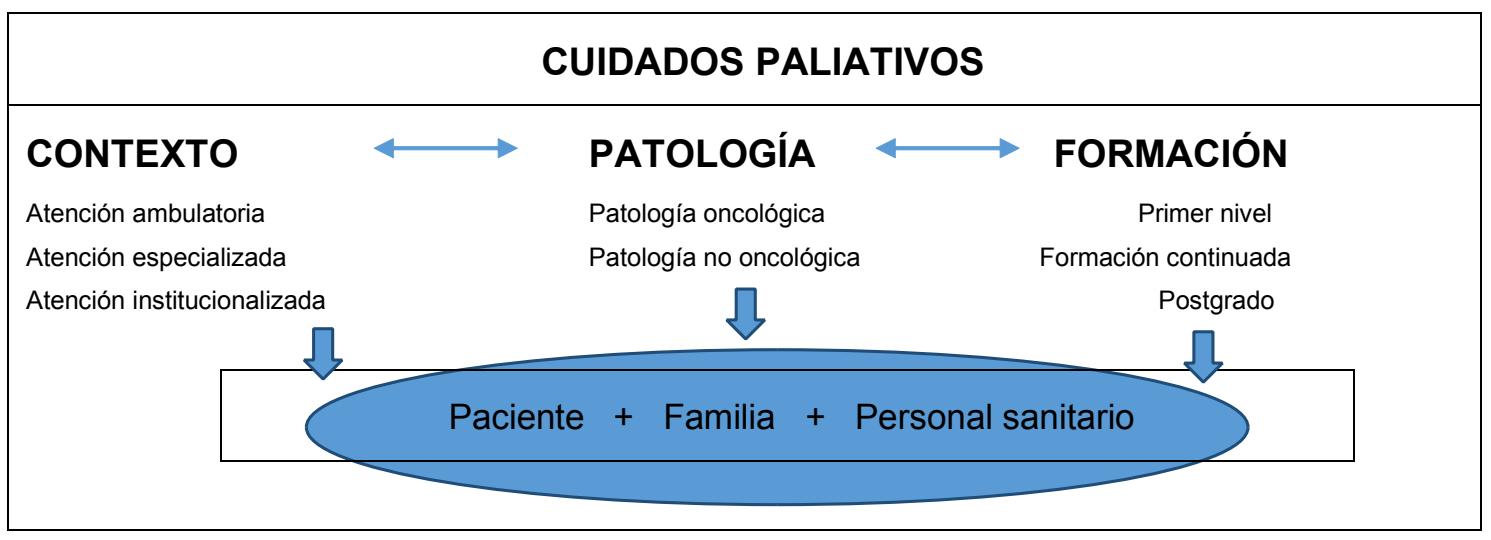

Tabla 1: Frecuencias y porcentajes sobre el nivel de competencias en función del lugar de origen.

Cuestionario FATCOD-S

Competencias muy negativas (30-60 puntos)

Competencias negativas (61-90 puntos)

Competencias positivas (91-120 puntos)

Competencias muy positivas (121-150 puntos)

\begin{tabular}{lll} 
& \multicolumn{2}{c}{ Los Ríos Valor-p } \\
$6(1,8 \%)$ & $1(0.9 \%)$ & 0.246 \\
$44(13,5 \%)$ & $12(10.5 \%)$ \\
$273(84 \%)$ & $98(86 \%)$ \\
$2(6 \%)$ & $3(2.6 \%)$
\end{tabular}


Tabla 2: Descripción de las variables sociodemográficas del estudio. Frecuencia, media global de la puntuación en la escala Frommelt y deviación estándar (Ds)

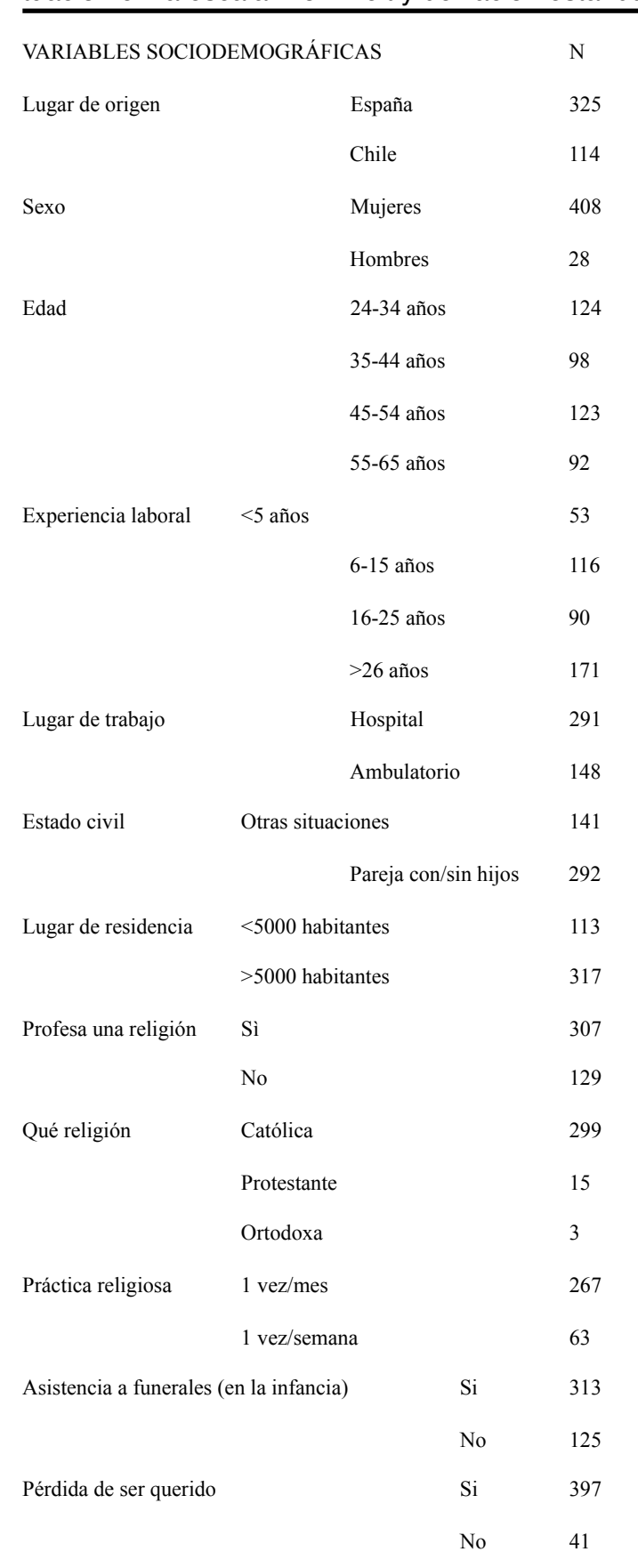

Media (Ds) $\quad$ Valor- $p$

$97.71(9.06) \quad 0.002$

$100.19(14.52)$

$98.52(11.25) \quad 0.558$

$98.44(9.4)$

$101.36(8.07) \quad 0.000$

$97.45(7.49)$

98.24 (10.98)

$95.30(18.05)$

$102.93(9.31) \quad 0.000$

98.64 (7.86)

$98.46(7.55)$

$96.72(14.85)$

$98.58(9.55) \quad 0.002$

98.37 (14.42)

$100.71(7.6) \quad 0.443$

97.48 (12.37)

$98.66(8.17) \quad 0.001$

$98.47(11.99)$

$98.79(11.19) \quad 0.001$

$89.16(3.48)$

$98.41(11.26) \quad 0.063$

$102.1(10.06)$

$97.66(6.5)$

$98.78(11.72)$

0.155

$97.48(8.71)$

$98.01(12.08) \quad 0.040$

$99.77(8.46)$

$98.51(11.5) \quad 0.046$

$98.64(6.38)$

Percepción sobre la preparación emocional para el cuidado terminal

$\begin{array}{ll}\text { Desde } 1 \text { a } 3 \text { puntos } & 142 \\ \text { Desde } 4 \text { a } 5 \text { puntos } & 288\end{array}$

$97.65(13.92) \quad 0.001$

$97.87(9.21)$

Percepción sobre la preparación técnica para el cuidado terminal

$\begin{array}{lll}\text { Desde } 1 \text { a } 3 \text { puntos } & 190 & 98.79(13.66) \\ \text { Desde } 4 \text { a } 5 \text { puntos } & 247 & 98.29(8.25)\end{array}$


Tabla 3: Estadísticos descriptivos de los enunciados relacionados con el factor I de la escala FATCOD-S según el lugar de origen.

\begin{tabular}{|c|c|c|c|}
\hline \multirow[t]{2}{*}{ Enunciados de la escala FATCOD-S } & Navarra & Los Rios & \multirow{2}{*}{$\begin{array}{c}\text { Valor } \\
\mathrm{p}\end{array}$} \\
\hline & $M(D s)$ & M (Ds) & \\
\hline Factor I & $41.84(6.35)$ & $\begin{array}{c}43.39 \\
(7.8)\end{array}$ & 0.063 \\
\hline 1.Proporcionar cuidados al moribundo es una experiencia que merece la pena & $4.46(0.8)$ & $4.6(0.8)$ & 0.025 \\
\hline 2.La muerte no es lo peor que le puede pasar a una persona & $4.17(1.2)$ & $4.41(1.0)$ & 0.007 \\
\hline 3.Es incómodo hablar sobre la muerte inminente con el moribundo & $3.61(1.1)$ & $3.39(1.2)$ & 0.088 \\
\hline 5.A mí no me gustaría atender a un moribundo & $2.03(1.1)$ & $1.75(1.0)$ & 0.021 \\
\hline $\begin{array}{l}\text { 6.Los cuidadores que no pertenezcan a la familia no deben ser los que hablen } \\
\text { de la muerte con el moribundo }\end{array}$ & $1.95(1.0)$ & $2.02(1.1)$ & 0.739 \\
\hline 7.El periodo de tiempo requerido para atender a un moribundo me frustraría & $1.96(1.0)$ & $2.29(1.2)$ & 0.012 \\
\hline $\begin{array}{l}\text { 8.Me disgustaría que el moribundo al que estoy atendiendo perdiera la } \\
\text { esperanza de recuperarse }\end{array}$ & $2.13(1.1)$ & $2.17(1.2)$ & 0.899 \\
\hline 9.Es difícil establecer una relación cercana con el moribundo & $2.4(1.1)$ & $2.46(1.2)$ & 0.761 \\
\hline $\begin{array}{l}\text { 11. Cuando un paciente pregunta ¿me estoy muriendo? creo que es mejor } \\
\text { cambiar de tema hacia algo más alegre }\end{array}$ & $1.62(0.9)$ & $1.98(1.1)$ & 0.001 \\
\hline $\begin{array}{l}\text { 13. Tendría esperanzas de que la persona a la que atiendo muriera cuando no } \\
\text { esté }\end{array}$ & $2.3(1.1)$ & $2.63(1.2)$ & 0.022 \\
\hline 14. Tengo miedo de trabar una amistad con el paciente & $2.21(1.1)$ & $2.17(1.3)$ & 0.283 \\
\hline 15.Sentiría ganas de huir cuando la persona por fin muriera & $1.9(1.0)$ & $1.66(0.9)$ & 0.008 \\
\hline $\begin{array}{l}\text { 17. Conforme el paciente se aproxima a la muerte, los cuidadores ajenos a la } \\
\text { familia deben retirar su implicación con el paciente }\end{array}$ & $1.91(1.0)$ & $2.27(1.3)$ & 0.024 \\
\hline $\begin{array}{l}\text { 26.Me sentiría incómodo si al entrar en la habitación de un enfermo terminal, le } \\
\text { encuentro llorando }\end{array}$ & $2.5(1.2)$ & $2.57(1.4)$ & 0.176 \\
\hline $\begin{array}{l}\text { 29.Los miembros de la familia que permanecen cerca del moribundo a menudo } \\
\text { interfieren en el trabajo de los profesionales con el paciente }\end{array}$ & $2.54(1.1)$ & $2.75(1.2)$ & 0.145 \\
\hline $\begin{array}{l}\text { 30.Es posible para los cuidadores ajenos a la familia ayudar a los pacientes a } \\
\text { preparar la muerte }\end{array}$ & $4.16(0.8)$ & $4.3(0.8)$ & 0.091 \\
\hline
\end{tabular}


Tabla 4: Estadísticos descriptivos de los enunciados relacionados con el factor II de la escala FATCOD-S según el lugar de origen.

\begin{tabular}{|c|c|c|c|}
\hline \multirow[t]{2}{*}{ Enunciados de la escala FATCOD-S } & Navarra & Los Ríos & \multirow[t]{2}{*}{ Valor $\mathrm{p}$} \\
\hline & M (Ds) & M (Ds) & \\
\hline Factor II & $\begin{array}{c}52.46 \\
(4.3)\end{array}$ & $\begin{array}{l}54.54 \\
(3.2)\end{array}$ & 0.000 \\
\hline $\begin{array}{l}\text { 4.Las atenciones a la familia del paciente deberían continuar a lo largo del } \\
\text { periodo de dolor y duelo }\end{array}$ & $4.61(0.7)$ & $4.86(0.4)$ & 0.000 \\
\hline 12.La familia debería estar involucrada en el cuidado físico del moribundo & $4.46(0.7)$ & $4.72(0.6)$ & 0.000 \\
\hline $\begin{array}{l}\text { 16. Las familias necesitan un soporte emocional para aceptar los cambios } \\
\text { de humor del moribundo }\end{array}$ & $4.47(0.7)$ & $4.77(0.5)$ & 0.000 \\
\hline $\begin{array}{l}\text { 18.Las familias deben preocuparse por ayudar a que el moribundo } \\
\text { aproveche lo que le queda de vida }\end{array}$ & $4.09(0.9)$ & $4.66(0.6)$ & 0.000 \\
\hline $\begin{array}{l}\text { 19.El moribundo no tiene permitido tomar decisiones sobre su cuidado } \\
\text { físico }\end{array}$ & $1.26(0.7)$ & $1.32(0.8)$ & 0.005 \\
\hline $\begin{array}{l}\text { 20.Las familias deben mantener un ambiente tan normal como sea posible } \\
\text { para el moribundo }\end{array}$ & $4.06(1.0)$ & $4.32(0.9)$ & 0.000 \\
\hline 21. Es beneficioso para el moribundo verbalizar sus sentimientos & $4.73(0.5)$ & $4.89(0.4)$ & 0.000 \\
\hline 22.Los cuidados deben extenderse hasta los familiares del moribundo & $4.72(0.5)$ & $4.9(0.3)$ & 0.000 \\
\hline $\begin{array}{l}\text { 23. Los cuidadores deben ofrecer a los moribundos un horario flexible de } \\
\text { visitas }\end{array}$ & $4.44(0.8)$ & $4.66(0.7)$ & 0.003 \\
\hline $\begin{array}{l}\text { 24.El moribundo y su familia deben ser los responsables de la toma de } \\
\text { decisiones }\end{array}$ & $4.58(0.6)$ & $4.72(0.5)$ & 0.008 \\
\hline $\begin{array}{l}\text { 25.La adicción a la medicación contra el dolor no debe preocupar cuando } \\
\text { se trata de un moribundo }\end{array}$ & $4.36(1.0)$ & $3.94(1.3)$ & 0.008 \\
\hline 27.Los moribundos deben recibir respuestas sinceras sobre su enfermedad & $4.45(0.8)$ & $4.72(0.5)$ & 0.002 \\
\hline $\begin{array}{l}\text { 28. La educación de las familias sobre la muerte no es una responsabilidad } \\
\text { de los cuidadores ajenos a la familia }\end{array}$ & $2.23(1.1)$ & $2.06(1.2)$ & 0.075 \\
\hline
\end{tabular}

Tabla 5. Análisis descriptivo y no paramétrico de los enunciados de la escala FATCOD-S según las variables sociodemográficas de la muestra global.

$\begin{array}{llll}\text { Variables sociodemográficas en relación con los ítems de la escala FATCOD-S } & \\ \text { Grupo total (N=439) } & \mathrm{M}(\mathrm{Ds}) & \mathrm{M}(\mathrm{Ds}) & \text { Valor } p \\ \text { SEXO } & \text { Femenino } & \text { Masculino } & \\ \text { Item 16. } & 1.87(1.0) & 1.71(0.9) & 0.018 \\ \text { LUGAR DE TRABAJO } & \text { Hospital } & \text { Ambulatorio } & \\ \text { Item 11. } & 1.76(1.0) & 1.67(0.9) & 0.052 \\ \text { Item 23. } & 4.63(0.7) & 4.48(0.7) & 0.014\end{array}$

ESTADO CIVIL

Otras situaciones Pareja con/sin hijos 
Item 4.

Item 7.

LUGAR DE RESIDENCIA

Item 3 .

Item 16.

PROFESA UNA RELIGIÓN

Item 2.

Item 23.

Fattore II

PRÁCTICA RELIGIOSA

Item 8 .

Item 19.

Item 22.

Item 30.

\section{ASISTENCIA FUNERALES INFANCIA}

Item 6.

PÉRDIDA DE UN SER QUERIDO

Item 2.

Item 23.

Item 25

EDAD

Item 3.

Item 5 .

Item 11.

Item 13.

Item 16.

Item 18.

Item 19.

Item 23.

Item 25.

\section{AÑOS EXPERIENCIA}

Item 4.

Item 11.

Item 12.

Item 13

Item 16.

Item 18

Item 23.

Item 25

\begin{abstract}
$4.77(0.5)$
\end{abstract}
$2.05(1.0)$

$4.63(0.7)$

$2.25(1.1)$

$>5000$ Habitantes

$3.72(1.1)$

$3.5(1.1)$

$4.58(0.6)$

NO

SI

$4.16(1.1)$

$4.59(0.7)$

$53.2(4.0)$

1/Mes

$2.08(1.1)$

$1.23(0.7)$

$4.84(0.3)$

$4.22(0.8)$

Sí

2.05 (1.1)

Sí

$4.13(1.1)$

$4.59(0.7)$

$4.24(1.2)$

No

No
0.054

0.024

0.017

0.049

$4.22(1.3) \quad 0.023$

$4.44(0.7) \quad 0.000$

$52.11(4.6) \quad 0.024$

1/Semana

$2.4(1.0) \quad 0.023$

$1.48(0.9) \quad 0.024$

$4.72(0.4) \quad 0.016$

$3.98(0.8) \quad 0.020$

$1.96(1.2) \quad 0.009$

$4.52(0.8) \quad 0.052$

$4.52(0.6) \quad 0.041$

$3.86(1.3) \quad 0.018$

\begin{tabular}{|c|c|c|c|c|}
\hline 24-34 años & 35-44 años & 45-54 años & \multicolumn{2}{|c|}{ 55-65 años } \\
\hline $3.47(1.1)$ & $3.39(1.2)$ & $3.8(1.0)$ & $3.55(1.2)$ & 0.021 \\
\hline $1.79(1.0)$ & $1.84(1.0)$ & $2.18(1.1)$ & $2.01(1.1)$ & 0.014 \\
\hline $1.94(1.1)$ & $1.64(0.8)$ & $1.52(0.8)$ & $1.72(1.0)$ & 0.006 \\
\hline $2.6(1.2)$ & $2.4(1.1)$ & $2.4(1.1)$ & $2.07(1.0)$ & 0.021 \\
\hline $4.57(0.5)$ & $4.7(0.6)$ & $4.54(0.6)$ & $4.34(0.9)$ & 0.006 \\
\hline $4.47(0.6)$ & $4.3(0.9)$ & $4.1(1.0)$ & $4.02(1.0)$ & 0.026 \\
\hline $1.24(0.7)$ & $1.25(0.8)$ & $1.15(0.3)$ & $1.44(0.9)$ & 0.009 \\
\hline $4.41(0.8)$ & $4.59(0.7)$ & $4.58(0.7)$ & $4.43(0.7)$ & 0.044 \\
\hline $3.87(1.3)$ & $4.33(1.0)$ & $4.42(1.1)$ & $4.44(1.0)$ & 0.000 \\
\hline$<5$ años & 6-15 años & 16-25 años & $>26$ años & \\
\hline $4.87(0.3)$ & $4.71(0.5)$ & $4.73(0.5)$ & $4.56(0.7)$ & 0.015 \\
\hline $2.15(1.2)$ & $1.77(0.9)$ & $1.53(0.8)$ & $1.62(0.9)$ & 0.004 \\
\hline $4.7(0.7)$ & $4.57(0.7)$ & $4.53(0.8)$ & $4.46(0.7)$ & 0.024 \\
\hline $2.7(1.3)$ & $2.55(1.1)$ & $2.24(1.1)$ & $2.27(1.1)$ & 0.046 \\
\hline $4.75(0.5)$ & $4.48(0.7)$ & $4.71(0.5)$ & $4.43(0.8)$ & 0.001 \\
\hline $4.72(0.5)$ & $4.34(0.7)$ & $4.26(0.9)$ & $4.01(1.1)$ & 0.000 \\
\hline $4.53(0.8)$ & $4.4(0.8)$ & $4.6(0.7)$ & $4.49(0.7)$ & 0.019 \\
\hline $3.55(1.4)$ & $4.16(1.1)$ & $4.29(1.2)$ & $4.5(1.0)$ & 0.000 \\
\hline
\end{tabular}


OSES-ZUBIRI - CASAS-FERnÁNDEZ DE TEJERINA - SEgUel-PALMA COMPETENCIAS EN FIN DE VIDA ENTRE UN GRUPO DE ENFERMEROS ESPAÑOLES Y CHILENOS PREPARACIÓN EMOCIONAL De 1 a 3 ptos De 4 a5 ptos

Factor I

PREPARACIÓN TÉCNICA

Factor I
$44.64(6.5)$

De 1 a 3 ptos
41.41 (6.6)

0.000 